\title{
Comparison of analgesic effects of programmed intermittent epidural bolus and continuous epidural infusion after total knee arthroplasty
}

\author{
Shinkyu Kang ${ }^{2}$, Sangyoon Jeon ${ }^{1}$, Ji Hyun Choe ${ }^{1}$, Si Ra Bang ${ }^{1}$, and Ki Hwa Lee ${ }^{1}$ \\ Department of Anesthesiology and Pain Medicine, ${ }^{1}$ Inje University College of Medicine, Haeundae Paik Hospital, ${ }^{2}$ Busan St. Mary \\ Hospital, Busan, Korea
}

After total knee arthroplasty (TKA), patients suffer pain; therefore, many analgesic techniques have been invented. Epidural analgesia is widely used and can be delivered by continuous epidural infusion (CEI), patient controlled epidural analgesia (PCEA), or intermittent epidural bolus (IEB). However, CEI is associated with greater incidence of motor block with higher concentrations of anesthetics, and inadequate analgesia is sometimes observed with diluted solutions [1]. One study suggested that uniform diffusion of anesthetics in epidural space is better obtained by administration of bolus [2], however, additional work is needed on nurses or patients.

The development of devices has allowed for delivery of bolus at regular intervals, so that programmed intermittent epidural bolus (PIEB) has become practicable. However, there is no consensus with regard to which technique is better, and comparative studies of analgesic effect after orthopedic surgery of lower extremities are rare. Hence, we compared the analgesic effect of CEI and PIEB.

After obtaining approval of the local ethics committee and written informed consent from all subjects, 53 American Society of Anesthesiologists 1, 2 patients who underwent TKA were recruited. Patients with anaphylaxis to bupivacaine and morphine, contraindications to spinal anesthesia were excluded. Randomization was achieved by block randomization.

The patient's circulation was preloaded with $6-10 \mathrm{ml} / \mathrm{kg}$ Hartmann's solution. The patient was placed in lateral position and a $25 \mathrm{G}$ spinal needle was introduced at L3-4 or L4-5 interspace; $12-14 \mathrm{mg}$ of $0.5 \%$ bupivacaine $\mathrm{HCl}$ heavy was injected intrathecally. An $18 \mathrm{G}$ epidural needle was then introduced in the same spot by loss of resistance technique. After identification of epidural space, a $20 \mathrm{G}$ epidural catheter was advanced $3 \mathrm{~cm}$ in the cephalad direction. Before surgery, pinprick test was performed to ensure that sensory block was above T10. At the end of surgery, all patients received $6 \mathrm{ml}$ of $0.125 \%$ bupivacaine + $0.005 \%$ morphine sulfate as a loading dose via an epidural catheter. The GemStar ${ }^{\mathrm{TM}}$ infusion system (Hospira ${ }^{\circledR}$, Lake Forest, IL, USA) delivered $3 \mathrm{ml}$ of $0.125 \%$ bupivacaine $+0.005 \%$ morphine sulfate hourly, beginning immediately after loading dose administration in the CEI group, but 60 minutes later in the PIEB group. We also checked that the extent of sensory block was above $\mathrm{T} 12$ at the start of analgesia.

Vital signs, numeric rating scale (NRS), modified bromage scale and side effects were assessed every $4 \mathrm{~h}$ by an independent trained observer. Hypotension was defined as a systolic blood pressure of less than $90 \mathrm{mmHg}$, bradycardia was defined as a heart rate of less than 50 beats/min, and respiratory depression was defined as a respiratory rate of less than 8 breaths $/ \mathrm{min}$. In cases of hypotension, ephedrine $10 \mathrm{mg}$ was administered intravenously. In cases of nausea and vomiting, ondansetron $4 \mathrm{mg}$ was administered intravenously. In cases of bradycardia, atropine $0.5 \mathrm{mg}$ was administered intravenously. In cases of respiratory depression, naloxone $200 \mu \mathrm{g}$ was administered intra-

Corresponding author: Sangyoon Jeon, M.D., Department of Anesthesiology and Pain Medicine, Inje University College of Medicine, Haeundae Paik Hospital, Jwa-4 dong, Haeundae-gu, Busan 612-896, Korea. Tel: 82-51-797-0424, Fax: 82-51-797-0499, E-mail: maestro@paik.ac.kr (c) This is an open-access article distributed under the terms of the Creative Commons Attribution Non-Commercial License (http:// creativecommons.org/licenses/by-nc/3.0/), which permits unrestricted non-commercial use, distribution, and reproduction in any medium, provided the original work is properly cited. 


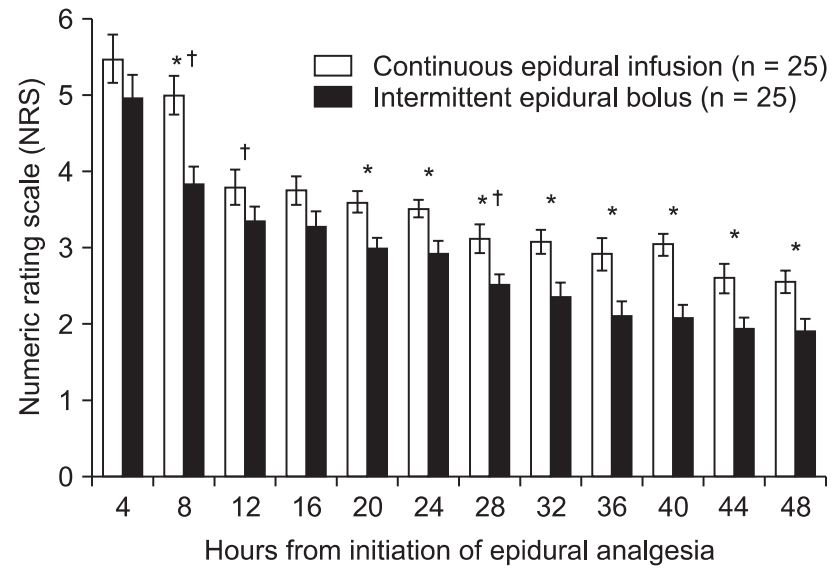

Fig. 1. Time course of Numeric rating scale (NRS) in Continuous epidural infusion (CEI) and Programmed intermittent epidural bolus (PIEB) groups. There have been significant decreases in NRS compared with the value at the initiation of epidural analgesia, and also significant difference between CEI and PIEB group at 8 hours, 20 hours and after. ${ }^{*} \mathrm{P}<0.05$ different between CEI and IEB groups at each time point by Mann-Whitney $\mathrm{U}$ test, ${ }^{\dagger} \mathrm{P}<0.05$ different from value of the previous time point by repeated measures ANOVA of both CEI and IEB groups.

venously. In cases of inadequate analgesia, pethidine $25 \mathrm{mg}$ was administered intravenously.

Data were evaluated using the Mann-Whitney U test for continuous variables and Fisher exact test for categorical variables. Repeated-measures ANOVA was used for comparison of effect NRS with time. Statistical analyses were performed using SPSS (Version 18.0, SPSS Inc., USA) and P values under 0.05 were considered statistically significant.

A total of 53 subjects were recruited, and three patients were excluded. The two groups did not differ in demographic data. Modified bromage scale was 2 or under during the entire study and there was no significant difference $(\mathrm{P}>0.05)$. According to repeated-measures ANOVA of both the CEI and PIEB groups, at 8,12 , and $28 \mathrm{~h}$, the value of NRS differed from the value of the previous time point $(\mathrm{P}<0.05)$; and, according to MannWhitney $U$ Test, significant difference was observed between the $\mathrm{CEI}$ and PIEB groups at $8,20 \mathrm{~h}$ and after $(\mathrm{P}<0.05)$. Decreases in NRS were observed in both groups; however, NRS of the PIEB group was significantly lower than that of the CEI group (Fig. 1). The number of rescue medicine usages per person was significantly lower in the PIEB group (PIEB: $1.8 \pm 0.9$, CEI: $3.0 \pm$ $1.9, \mathrm{P}=0.001)$. No difference in side effects was observed.

The fact that the number of rescue medicine usages and NRS with time was lower in the PIEB group indicates that PIEB has better analgesic effects. According to one study [2], distribution of solution in epidural space is nonuniform. Outside of accumulations near sites of injection, no large channels of spread are apparent. Rather, solution appears to progress through multiple crevices in a path determined by structures of epidural space. In addition, distribution of anesthetics within epidural space is shared between the principal sites competing for uptake. These are mainly nerves, their covering, fat, and blood vessels. Bolus may create more pressure so anesthetics spread further though blockade and absorption. This can explain why PIEB has a better analgesic effect than CEI. Virmani et al. [3] reported that IEB has more fluctuation, so that CEI is appropriate. However, in that study, the interval of boluses was $3 \mathrm{~h}$, which could disturb the constant analgesic effect of IEB.

PCEA does not require a specific pump; there is a limitation in that anesthetics cannot be injected without patient's pain. Pain occurs after diminution of epidural blockade. If diminution of epidural blockade occurs, sensory neural input to the spinal cord has been shown to accelerate the decline of the block [4]. If this occurs, a larger dose of anesthetics is required to restore analgesia. However, PIEB provides anesthetics at appointed intervals and doses; therefore, we expect a more constant analgesic effect than with PCEA. In addition, because patients' involvement with PIEB is hard, PIEB has less risk of misuse.

In conclusion, no difference was observed in motor blockade and side effects, and NRS and rescue medicine usages were significantly lower in the PIEB group. Therefore, we conclude that PIEB has a superior analgesic effect, compared with CEI in analgesia of total knee arthroplasty, and various regimens may be developed.

\section{References}

1. Gambling DR, Yu P, Cole C, McMorland GH, Palmer L. A comparative study of patient controlled epidural analgesia (PCEA) and continuous infusion epidural analgesia (CIEA) during labour. Can J Anaesth 1988; 35: 249-54.

2. Hogan Q. Distribution of solution in the epidural space: examination by cryomicrotome section. Reg Anesth Pain Med 2002; 27: 150-6.

3. Virmani R, Ghai A, SinghA DK. Study to compare continuous epidural infusion and intermittent bolus of bupivacaine for postoperative analgesia following renal surgery. SAJAA 2008; 14: 19-22.

4. Ueda W, Sagara Y, Hirakawa M. Role of afferent neural input in regression of sensory paralysis during epidural analgesia. Anesth Analg 1992; 74: 358-61. 